\title{
Blinded by data
}

\section{The risks of the implicit focus on data in ICT for Sustainability}

\author{
${ }^{1 .}$ Centre for Sustainable Communications \\ KTH Royal Institute of Technology \\ Stockholm, Sweden
}

Jorge Luis Zapico ${ }^{1,2}$

zapico@kth.se
2. Media Technology
Linnaeus University
Växjö, Sweden

\begin{abstract}
Sustainability is a normative concept, building on ideas such as justice, equity and responsibility, and based on human culture and society. Computers, internet, and the technologies that are central in our network society embed also normative values and are part of a cultural context. But the work looking at computer technologies and sustainability has been mostly oriented towards either calculating the impact of technology or using technology as a tool for solving practical problems. ICT is seen as a neutral system to be used or study, while the more normative aspects are mostly overlooked. This paper explores some of the problems arising from these overlooked normative values, such as focusing only on quantifiable problems while forgetting other aspects that may be as important but not easy to put in numbers, or trusting too much in numbers while hiding assumption and model choices. This paper suggests that more critical reflection on these questions is needed in the research area, as well as developing more connections with existing research on these topics in more traditional disciplines.
\end{abstract}

Index Terms-ICT4S, data, risks, sustainability, uncertainties, rebound effects.

\section{INTRODUCTION}

Sustainability is a normative concept, building on ideas such as justice, equity and responsibility, and based on human culture and society. The most cited definition comes from the Brundtland's report for the UN World Commission on Environment and Development: "development that meets the needs of the present without compromising the ability of future generations to meet their needs" [1]. The key point in this definition is bringing together environmental and humanitarian concerns under the concept of equity (between and within generations). It includes the concept of needs and human wellbeing, in particular focusing on the world's poor, and the idea of limits on the environment ability to meet these needs. It frames the environmental problems not as something external to human society, but as a basis for our future. It includes the realization that the social and economic systems are not independent of the environment, but embedded in it, and that these systems have to take into account that we live on a limited planet. Sustainability is different than most other topics in science and engineering and can be defined as a "wicked problem" [2]. Wicked problems are problems that contain paradoxes, conflicts, changing requirement, complex interdependencies and that cannot be easily modeled by traditional scientific approaches [3]. Sustainability research and practice makes extensive use of modeling tools and quantitative techniques such as life cycle assessment (LCA) [4] that may seem less normative, but that embed also political ideological and ethical values [5].

ICT for sustainability (ICT4S) is a growing research area exploring the use of information technologies with a sustainability purpose [6]. Most ICT4S research has focused either on understanding the negative direct impacts of hardware such as energy use of internet $[7,8,9]$ and the generation of ewaste [10], or on the applications of using the technologies with a sustainability purpose, such as increasing the efficiency of systems and increasing dematerialization [11] or triggering behavioral change [12]. Computer technologies are treated either as a system to be understood, or as tools that can be used for some purpose. This paper argues that this focus on computers as tools, without reflecting upon the bias coming from their cultural context and history, creates problems and blindspots that are worth bringing attention to. First the paper presents the focus on quantitative data, central to the affordances provided by computer technologies, as a key component of ICT4S. Second, it discusses new characteristics and approaches that ICT is adding to how we work with sustainability work. Based on these, this paper explores some of the risks and blindspots of the focus on metrics and quantitative data in the context of a wicked normative problem such as sustainability. Finally, some proposals for contributions and further research in this topic are proposed.

\section{IT'S ALL ABOUT THE DATA}

It can be argued that the applications and research in the area of ICT for Sustainability are for the most part based on the creation, processing and communication of quantitative sustainability data [13]. This is not unexpected, as logic and arithmetic calculations are historically main tasks computers were created for. This text presents efficiency and behavioral change as two main topics of ICT4S that represent this focus on quantitative data. Efficiency is one of the key concepts that are heavily mentioned in the literature of ICT for Sustainability [14]. Reports such as Smart2020 [11], and Greener and Smarter [15], point out efficiency as the most important contribution that ICT can make to sustainability, and even as a central part 
in achieving a more sustainable society in general. In the context of ICT4S, efficiency usually means the use of ICT for optimizing a system or a process with a sustainability purpose, for instance reducing the amount of energy and materials required for delivering a service or the greenhouse gases produced when creating a product. ICT has always been used to increase efficiency, specially time-efficiency, accelerating existing processes [16]. This acceleration is the defining characteristic of the impact of ICT in society, and as Castells [17] argues, one of the pillars of the information society. The current discourse in ICT4S uses the terms smart or smarter, arguing that by measuring and controlling parameters, ICT can tune and automate existing systems, reducing emissions and resource consumption while maintaining or increasing utility. Examples of this include the topics of smart cities, smart grids, smart appliances, intelligent transport systems and smart logistics. Smart grid is representative for how ICT is being applied for sustainability related efficiency. Information technologies are used to improve the actual electricity grid system, for instance routing power more efficiently, reducing the need for excess capacity and allowing two-way real time information exchange between producers and customers for real time demand side management. Smart grid has been suggested as the single most important application of ICT for reducing greenhouse gas emissions [11]. Intelligent transport systems (ITS) is also a very active research area where the optimization potential of ICT is being explored, including the optimization of storage and transportation of goods [11]. ICT applications that are suggested to increase efficiency include inventory reduction, eco-driving, route optimization and intermodal shifts. A particular example is the computer models used by Japan National Institute of Informatics for calculating the best possible delivery route between different stores and providers, creating both economical and environmental savings [18]. Efficiency measures are also being applied to other fields, such as agriculture. For instance ICT based smart irrigation systems to reduce water usage and carbon emissions by context-aware watering schedules, using weather data and/or soil moisture and evapotranspiration sensing [19].

Another central theme of ICT for sustainability is the use of technology for supporting or triggering behavioral change towards sustainability. This includes research on eco-feedback, persuasive technologies, ambient awareness and pervasive and participatory sensing. This kind of research is most prominent in the area of Sustainable HCI. Di Salvo et al [12] in their survey of the research area found that $70 \%$ of the publication corpus on sustainable HCI was on persuasive and ambient awareness. A main concept in research about behavioral change is using technology for "making the invisible visible". Information technology is used to visualize and communicate data that is relevant for sustainability such as energy use, water use or carbon dioxide emissions [20]. This is seen as a precondition for acting and increasing sustainability: if it's visible, it's actionable and then it can be made sustainable [21]. Some examples of applications and research looking at these topics include: research on energy meters for visualizing energy consumption at home; the use of competition and other persuasive techniques for reducing energy consumption; projects making energy visible, such as transparent screens showing a metaphorical tree representing energy consumption, power cords that make electricity consumption visible, and lamps that reward low energy use [22,23]; visualization of water usage at home using a LED lights display in the shower [24]; visualization of carbon dioxide impact in the supply chain of products [25].

Many efficiency and behavioral change applications are based on the gathering, processing and visualization of metrics. Efficiency and smart solutions use ICT for gathering information, processing it and analyzing more optimal solutions, automating processes based on the data, and getting feedback from the changes. Behavioral change applications are in many cases (not all) based on providing quantitative feedback (either directly or in some persuasive way such as in games) to the users to help them make better decisions and increase their awareness about the impact of their actions. Using ICT for efficiency and behavioral change provides opportunities and solutions that can have a positive impact in sustainability. It could even be said that they are necessary. It is difficult to change something if there is no feedback of the starting state and of how the actions performed affect it. But the implicit emphasis on "what can be measured" creates problems and blindspots that are worth discussing, both for minimizing the risks and for opening up new opportunities that may be hidden.

\section{NEW DATA APPROACHES}

The use of new information technologies in quantitative data also adds special characteristics that differ from the way "traditional" methods in sustainability information, in practices such as LCA and environmental impact analysis. These characteristics show how the application of new technologies and approaches from ICT can transform (and is already transforming) how data is created, shared and visualized in sustainability practice $[13,26,27]$ :

\section{A. Bottom up automatic data}

The use of new technologies such as pervasive sensing, machine-readable data, and open licenses, together with new approaches such as crowdsourcing and mashups, are changing how data is created and collected. These approaches can change how sustainability data is created, from a mostly manual way to a more automated and bottom-up approach, where bigger amounts of data can be processed in a more programmatic style. With the exponential increase on the world's capacity to store, communicate and compute information and the increase of data availability connected to the physical world, the same approach could be applied to other products and services.

Crowdsourcing is also relevant in this context, as it changes the way data can be collected to a more social bottom-up approach. Relevant examples using crowdsourcing in a sustainability context include the gathering of weather data 
[28], the track of deforestation using satellite pictures ${ }^{1}$, or information about supply chains [25]. Pervasive sensing is another technology that can enable the automatic gathering of data. Examples of sustainability-oriented projects exploring this are: PEIR, an application from CENS at UCLA that tracks the user's location and movements using GPS and calculates the $\mathrm{CO}_{2}$ impact and the exposure to air pollution [29], and Air Quality $\mathrm{Egg}^{2}$, an open source crowdfunded sensor that can track $\mathrm{NO}_{2}$ and $\mathrm{CO}$ concentrations as indicators of air pollution.

\section{B. Real time and dynamic feedback}

Other important characteristic is the use of real time and dynamic feedback that is up to date with the latest data available. This is in contrast with existing sustainability approaches, such as LCA, where environmental information is mainly collected either in a static, retroactive process or in a prognosis, scenario based approach. Smart energy meters, that allow real time feedback of electricity consumption, are a representative example of this shift towards real time, and they are one of the key technologies used in many ICT4S behavioral change applications such as in ambient awareness projects $[20,30]$. This acceleration of time is one of the main characteristics of ICT and its impact in society [17].

\section{Transparency and openness}

Much of the existing sustainability data is published in commercial databases or expensive journal articles where the public does not have access to it, and/or written in paper or in a text document where a computer service cannot access it. Applying open licenses and machine-readable formats to sustainability data can make the information more accessible to the general public, and the machine-readability makes possible to easily reuse existing data fostering new innovations. There are initiatives working in this direction. Examples include Earthster ${ }^{3}$, an upcoming LCA tool with focus on open source and visualization, $\mathrm{AMEE}^{4}$ a UK company that provide environmental data in a machinereadable API, ELCD ${ }^{5}$, a European LCA database that is accessible for free in machine-readable XML format, and OpenLCA, an open source LCA modeling software [31].

\section{Dynamic visualization}

New technologies are increasing the possibilities of making the visualization more dynamic and interactive. Applications such as Webenergy ${ }^{6}, H_{\text {HelsinkiCO2 }}^{7}$, and Carbon.to [32], explore this space, allowing users to interact with environmental impact analysis, letting the users to change parameters of the impact assessment and play with the results,

\author{
http://forestwatchers.net/ \\ http://airqualityegg.com/ \\ http://www.earthster.org/ \\ https://www.amee.com/pages/api \\ http://lca.jrc.ec.europa.eu/lcainfohub/html/datasetsLicense.html \\ http://sympact.cs.bris.ac.uk/webenergy/main/start \\ http://helsinkico2.com
}

explore future scenarios, or use representations for better communication of quantitative information.

\section{E. User-driven innovation}

The affordances of information technologies are broadening innovation [33], and innovation is now driven not only by professionals, but also by end users and amateurs. In the case of sustainability practice, the increasing availability of data in open and machine-readable formats makes it possible to visualize, transform, and remix different datasets, and to generate new innovative usages outside the sustainability practice community [34]. Another key difference of this democratization of innovation is the openness of the process, as user innovations are usually freely revealed [33], which makes them easier to spread and to be adopted and adapted.

\section{DATA BLINDNESS}

The focus on quantitative data together with the transformative ways of working with data using new information technologies create new risks and challenges, which I group as "data blindness", or the risk of trusting only or too much in data.

\section{A. Trusting only on data}

While we have this focus on quantitative data, we have to remember that sustainability is a "wicked problem", not easily reduced to numbers and explicitly normative. One side of this problem is that a data-driven approach focuses de-facto on parts of sustainability that are possible or easy to quantify, while other areas that are more challenging to put numbers on are neglected. This can be seen in the focus on energy and $\mathrm{CO}_{2}$ emissions in many studies, for instance in the majority of life cycle assessments of ICT [7,35], while other problems such as toxicity do not get the same attention. This can also be argued in the case of behavioral change interventions, the focus on what can be measured means that there is an overrepresentation of projects working for instance with electricity consumption, while other areas that may be bigger but difficult to calculate, such as the impact of the production of consumer goods, are not being explored.

The focus on quantitative data and real-time information can create also a kind of time-shortsightedness. The quantitative nature of the data makes it difficult to look at longterm developments concerning the future, as there is no measured data for it. Höjer et al. [36] argue that this is a common problem for environmental system analysis tools, and that there is a need for more prospective thinking and integrating with future studies. The real-time focus of ICT can accentuate this problem, as data becomes more immediate and there is less room for reflection than in traditional environmental system analysis tools.

Brynjarsdóttir et al. [37] discuss some of these problems in the context of sustainable HCI, connecting them to the culture of modernism, which "trust that formal, rational methods capture essentially everything that matters about a given situation". They argue that this axiom of modernism is central to many of the persuasion and behavior change projects in sustainable $\mathrm{HCI}$, and that it narrows the view of sustainability 
assuming that the users are individuals maximizing utility based on information. They suggest that there should be a shift to more reflection instead of prescription, and to a focus on social and cultural practices instead of on individuals.

\section{B. Trusting too much on data}

Another problem of the focus on quantifiable data is how to deal with uncertainty and assumptions. Data such as environmental impact analysis or carbon footprints include many assumptions and modeled data, it has uncertainties and it may not be relevant for the specific case at hand. This is challenging to present and the quantitative data may lead to a false sense of exactness, as it is argued by Koomey et al [38]. These uncertainties are not a specific problem of ICT4S, but a general problem in environmental system analysis such as LCAs, related to poor data quality, use of aggregated data and wrong assumptions [39]. The assumptions in the model can also be influenced by the creators' values, while these choices remain mostly invisible to the users of the model. A classical example used is how the game SimCity simulates taxes so lowtaxes systems work better than more welfare oriented, thus communicating these values to the game users as they learn by simulation [40]. This blindness to the shortcoming of modeling tools is connected to the modernity values of calculability and predictability, the belief that everything can be numerically computed and that we can know beforehand the outcome of actions [41], in contrast with the wicked nature of sustainability, which includes paradoxes, complex interdependencies and changing requirements.

The problem with uncertainties and model assumption is accentuated when using computer technologies that use automatic calculation, crowdsourcing, or mashup and lack the textual space for analysis and discussion available in LCAs published as scientific articles. This produces new challenges that will need to be explored on how to work, calculate and communicate the uncertainties in the results. An example can be seen in Greenalytics, a web application that uses machinereadable data and APIs to calculate dynamically the carbon footprint of websites using a mashup approach [42]. It provides at first sight a very accurate result in kilograms or tons of $\mathrm{CO}_{2}$ with decimal points. It includes a disclaimer, explaining the uncertainties and how the result is not an accurate value but a best approximation based on the existing data. The details of how the numbers are calculated and the assumptions made are also presented. But this result includes many uncertainties and assumptions, and communicating these is a challenge [42].

\section{Trusting in the wrong data}

Another facet if the same problem is trusting in the wrong data. Models are imperfect, and when an impact is measured and optimized there might be rebound effects outside the boundaries of the model. When increasing the efficiency of a system, metrics are used to measure the efficiency, but this means that it is only the measured metric that will be optimized, even if the result for the end goal can be actually negative. Hilty et al. [43] explores this problem with efficiency, meaning that the efficiency gains of using ICT have been eaten up by rebound effects resulting in increasing output, not in reducing input. These rebound effects can be created by the choice of what is measured, in the case of efficiency it is usually the ratio not the total amount. A rebound effect is the lost gains in efficiency of technological progress due to that cost reduction leads to increased consumption of the same service, and/or increase in other spending due to higher income and systemic macro-economic effects [44]. This is not exclusive to ICT, but endemic to efficiency. A classical example is provided by Allenby [45], where he shows how cars have increased in fuel efficiency, but the total impact of cars have grown as cars are bigger and faster, there are many more cars and we drive them more. Hilty argues that using ICT for efficiency measures is not an enough precondition for saving resources, but that sufficiency measures, focusing on the total amount that is needed instead of on the efficiency ratio [46], and restricting input and output are needed in order to avoid these unwanted rebound effects [45].

\section{REFLECTIONS}

A focus of ICT4S research in quantitative data comes naturally from the affordances provided by ICT and by the implicit values of modernity. ICT have developed from computing tools and have transformed the way we can manage quantitative information, allowing the processing and storage of amounts of data that were unthinkable before. The values of modernity and informationalism focus also on the use models and metrics as a way, or the way, of understanding and controlling systems and nature. The use of quantitative data in ICT4S is a powerful tool, improving sustainability work by making variable visible and creating feedback mechanisms, and provides many opportunities for increasing efficiency of systems, measuring change, providing feedback on behavioral and policy changes, etc.

ICT can be used for sustainability practice, showing new ways of how sustainability data and information can be created, shared and visualized through the application of new technologies and paradigms, such as APIs, open licenses, crowdsourcing, pervasive sensors and mashups. These technologies add new affordances and transform the way of working with sustainability data:

- There is a focus on providing real time and dynamic feedback instead of using past static data.

- The data is gathered in an automatic way from the bottom up, instead than top-down approaches, using sensors, mashups and crowdsourcing.

- There is a focus on transparency and openness.

- Data is visualized dynamically and interactively.

- The innovation model is more open and broadened outside the sustainability field.

The mostly implicit focus in quantitative data in ICT4S, together with these new ways this data is created and communicated create risks and missed opportunities that need to be explored, what I call data blindness. This can mean that we trust only in data, focusing on problems and areas that are possible to model, while leaving aside other aspects that are difficult or impossible to quantify, and that we trust too much in data, forgetting that behind models and calculations there are 
assumptions and uncertainties, or that we may be measuring the wrong things. This does not mean to oppose to the use of quantitative data in ICT4S, but it suggests that more critical reflection on these questions is needed in the research area. I will present some of the possible action points:

- Developing more connections with existing research on these topics in more traditional disciplines. The limitations and risks of quantitative data in sustainability have been pointed out before in sustainability research. Finnveden [47], for instance, discusses the limitations in LCAs and other environmental system analysis tools and how data gaps, uncertainties in data and methodology, and the values embedded, make LCAs limited to provide prescription, for instance in deciding if a product is better than another one. It is suggested that environmental analysis can be a necessary and useful input in decision making, but more as a tool for learning, helping to identify critical points and in general to increase the knowledge of the studied systems. There is space for ICT4S research to expand the connections with sustainability research from other disciplines to gain insight about what it has been done already regarding these risks.

- Distinguishing models as tools and models as goals: When faced with uncertainties in modeling sustainability, we can increase the quality of the parameters, of the data collected, make it more real time, but in the end we still have a model, or as Borges wrote in on exactitude in Science, "becomes so exact that only a map on the same scale as the empire itself will suffice" [48]. I will argue that it is necessary to remind us that models and metrics are useful tools but in the end only proxies for working towards valuebased human-centered sustainability. In the case of efficiency, a similar effect can be observed, where the efficiency rate, from being a tool, becomes a goal in itself. There is a risk for rebound effects where efficiency (what is measured) improves, while the total impact (the original goal) increases instead of decrease.

- Increasing transparency: An area where ICT can make a difference for mitigating data-blindness is increasing the transparency and openness of sustainability data and studies. Much of the existing sustainability data is published in commercial databases or expensive journal articles where the public does not have access to it, and/or written in paper or in a text document where a computer service cannot access it. Applying open licenses to sustainability data (and models) can make the information more accessible, easier to replicate, and make assumptions visible to examine.

- Exploring new qualitative areas and research methods. A blindspot that is worth exploring are the applications of ICT for areas of sustainability and environmental impacts that are difficult to quantify and have therefore been most forgotten in research. This could include, as Brynjarsdottir et al [37] suggest, a focus in reflection not in prescription, and a shift from behaviors to practices, going beyond the focus on individual actions and metrics towards more holistic society practices. This type of more qualitative research will create also methodological challenges and call for more interdisciplinary collaborations in ICT4S.

\section{CONCLUSIONS}

"Grownups love numbers. When you tell them that you have made a new friend, they never ask you any questions about essential matters. They never say to you, "What does his voice sound like? What games does he love best? Does he collect butterflies?" Instead, they demand: "How old is he? How many brothers does he have? How much does he weigh? How much money does his father make?" Only from these figures do they think they have learned anything about him. [...] But certainly, for us who understand life, figures are a matter of indifference." - Le Petit Prince [49]

Empowering new ways of creating, processing and communicating sustainability data is and will be a main topic of ICT4S research, connected to some of the most explored parts of the research field such as using technology for increasing efficiency and for providing feedback and facilitating behavior change. These are powerful ways in which ICT can contribute to sustainability work. But there is a need for more discussion and reflection on the values and assumptions behind the focus on quantitative data, making it more explicit instead than being tacit. If not, we risk the presented problems of trusting only and/or too much in data or focusing on the wrong data and hiding rebound effects. It is especially important to identify and work with these specific problems and risks when applying new ICT approaches that transform how we work with data using mashup approaches, sensors, real time data, etc. This paper tried to open a bit more this discussion and proposes some action points, such as increasing the connection to existing research on traditional areas of sustainability research that have a longer tradition on dealing with the limitation of quantitative data and models in sustainability, to use the affordances of ICT to increase the transparency of data and models, and to have a wider perspective and explore possible blindspots of relevant topics and research methods that we may be missing out on, and that could enrich the ICT4S field.

\section{COPYRIGHT FORMS}

Released in Creative Commons BY http://creativecommons.org/licenses/by/3.0/ 


\section{ACKNOWLEDGMENT}

This paper is based on the $\mathrm{PhD}$ thesis "Hacking for Sustainability" at the KTH Centre for Sustinable Communications [46].

\section{REFERENCES}

[1] Brundtland, G.H. (1987). Our Common Future. United Nations World Commission on Environment and Development. Oxford University Press, Oxford.

[2] Levin, K., B. Cashore, S. Bernstein and G. Auld. (2009). Playing it forward: Path dependency, progressive incrementalism, and the "Super Wicked" problem of global climate change. IOP Conference Series: Earth and Environmental Science 50 (6). Bibcode 2009E\&ES....6X2002L. Doi:10.1088/17551307/6/50/502002.

[3] Rittel, H. Webber, M. (1973). Dilemmas in a general theory of planning. Policy Sciences vol:4(2)

[4] Finkbeiner, M., Inaba, A., Tan, R., Christiansen, K., \& Klüppel, H. J. (2006). The new international standards for life cycle assessment: ISO 14040 and ISO 14044. The international journal of life cycle assessment, 11(2), 80-85.

[5] Finnveden, G. (1997). Valuation Methods Within LCA - Where are the Values? International Journal of LCA 2(3) 163-169

[6] Hilty, L.M. Lohmann, W. Huang, E.M. (2011). Sustainability and ICT - An overview of the field . Notizie di POLITEIA, XXVII, 104, 13-28.

[7] Arushanyan, Y. Ekener-Petersen, E. Finnveden, G. (2013). Review: Lessons learned - Review of LCAs for ICT products and services. Comput. Ind. 65, 2 (February 2014), 211-234. DOI $=10.1016 /$ j.compind.2013.10.003

[8] Malmodin, J., Moberg, Å., Lundén, D., Finnveden, G., \& Lövehagen, N. (2010). Greenhouse gas emissions and operational electricity use in the ICT and entertainment \& media sectors. Journal of Industrial Ecology, 14(5), 770-790.

[9] Weber, C. L., Koomey, J. G., and Matthews, H. S. (2010a). The energy and climate implications of different music delivery methods. Journal of Industrial Ecology. Vol:14(5) , DOI: 10.1111/j.1530-9290.2010.00269x

[10] Shakila Umair, Anna Björklund och Elisabeth Ekener Petersen, Social Life Cycle Inventory and Impact Assessment of Informal recycling of Electronic ICT Waste in Pakistan, ICT4S 2013, pp. 52-58, International Conference on Information and Communication Technologies for Sustainability

[11] Climate Group, The. (2008). Smart2020: Enabling the low carbon economy in the information age. GeSI (Global esustainability

initiative) http://www.theclimategroup.org/assets/resources/publications/S mart2020Report.pdf

[12] DiSalvo, C., Sengers, P., and Brynjarsdóttir, H. (2010). Mapping the landscape of sustainable HCI. In Proceedings of the 28th international conference on Human factors in computing systems (pp. 1975-1984). ACM.

[13] Zapico, J. L., N. Brandt. and M. Turpeinen. (2010). Environmental Metrics. Journal of Industrial Ecology, Vol:14(5) no. doi: 10.1111/j.1530-9290.2010.00272.x

[14] Hilty, L. M. (2008). Information technology and sustainability. BoD-Books on Demand.

[15] OECD. (2010). Greener and Smarter - ICTs, the Environment and Climate Change. http://www.oecd.org/dataoecd/27/12/45983022.pdf
[16] Hilty, L.M. Behrendt, S. Binswanger, M. Bruinink, A. Erdmann, L. Freohlich, J. Koehler, A. Kuster, N. Som, C. and Wuertenberger, F. (2005). The precautionary principle in the information society - Second edition. Report of the Centre for Technology Assessment. TA SWISS.

[17] Castells, M. (1996). The Rise of the Network Society. (The Information Age: Economy, Society and Culture. Volume I). Blackwell Publishers.

[18] Satoh, Ichiro. (2008). A Specification Framework for Earthfriendly Logistics. Proceedings of 28th IFIP WG6.1 International Conference on Formal Techniques for Networked and Distributed Systems (FORTE'2008), Lecture Notes in Computer Science (LNCS), vol.5048, pp.251-266, Springer, June 2008.

[19] Mutchek, M.A., and E.D. Williams. (2010). Design space characterization for meeting costs and carbon reduction goals: smart irrigation controllers in the southwestern United States. Journal of Industrial Ecology 14(5). DOI: 10.1111/j.15309290.2010.00282x

[20] Froehlich, J. Findlater, L. and Landay, J. (2010). The Design of Eco-Feedback Technology. CHI 2010, Atlanta, Georgia, USA. ACM.

[21] Bonanni, L. Busse, D.K. Thomas, J.C. Blevis, E. Turpeinen, M. Jardim Nunes, N. (2011). Visible - actionable - sustainable: sustainable interaction design in professional domains. In Proceeding CHI EA '11 CHI'11 Extended Abstracts on Human Factors in Computing Systems, ACM. NY, USA

[22] Broms, L. Ilstedt Hjelm, S and Bång, M. (2008). Persuasive Engagement: Exploiting lifestyle as a driving force to promote energy-aware use patterns and behaviours. Proceedings of DRS2008, Design Research Society Biennial Conference, Sheffield, UK July 16-19 2008.

[23] Mazé, R. Redström, J. (2008). Switch! Energy ecologies in everyday life. International Journal of Design, 2(3): 55-70.

[24] Kappel, K. and Grechenig, T. 2009. "show-me": Water Consumption at a glance to promote Water Conservation in the Shower. The 4th International Conference on Persuasive Technology.

[25] Bonanni, L et al. (2010). Small business applications of sourcemap. Proceedings of CHI '10. Atlanta, Georgia, USA, 2010. 937. c2010 ACM.

[26] Davis, C., I. Nikolic, and G.P.J. Dijkema. (2010). Industrial Ecology 2.0. Journal of Industrial Ecology, Vol:14(5) no. doi: 10.1111/j.1530-9290.2010.00281.x

[27] Zapico, J.L (2014). Hacking for Sustainability. Doctoral dissertation in Media Technology and Graphic Arts. KTH Royal Institute of Technology.

[28] Elevant, K. Turpeinen, M. (2011). Improving Weather and Climatic Information Quality with User-generated observations. In Proceeding of International conference of System Sciences (HICSS), 2011 44th Hawaii.

[29] Agapie, E. Chen, G. Houston, D. Howard, E. Kim, J. Mun, M.Y. Mondschein, A. Reddy, S. Rosario, R. Ryder, J. Steiner, A. Burke, J. Estrin, E. Hansen, M.. Rahimi, M. (2008). Seeing Our Signals: Combining location traces and web-based models for personal discovery. Ninth Workshop on Mobile Computing Systems and Applications (HotMobile), February 25-26, 2008.

[30] Riche, Y. Dodge, J. Metoyer, R.A. (2010). Studying always-on electricity feedback in the home. In CHI 2010: Home eco behavior. CHI 2010 Atlanta, GA, USA. 
[31] Ciroth, A. (1997). OpenLCA - a new open source software for Life Cycle Assessment. International Journal of LCA 12(4) 209-210.

[32] Zapico, J.L. Turpeinen, M. Guath, M. (2011). Kilograms or cups of tea: Comparing footprints for better $\mathrm{CO}_{2}$ understanding. In PsychNology Journal 9(1), pp.43-54.

[33] Von Hippel, E. (2005). Democratizing innovation. MIT Press. Cambridge, MA

[34] Zapico, J.L. Pargman, D. Ebner, H. Eriksson, E. (2013). Hacking sustainability: Broadening participation through Green Hackathons. International Workshop on EUD for Supporting Sustainability in Maker Communities. IS EUD 2013, June 11-13, 2013, IT University of Copenhagen, DK.

[35] Teehan, P. and Kandlikar, M. (2012). Sources of Variation in Life Cycle Assessments of Desktop Computers. Journal of Industrial Ecology 16,182-S194.

[36] Höjer, M. Ahlroth, S. Dreborg, K.H. Ekvall, T. Finnveden, G. Hjelm, O. Hochschorner, E. Nilsson, M. and Palm, V. (2008). Scenarios in selected tools for environmental system analysis. Journal of Cleaner Production 16, 1958-1970.

[37] Brynjarsdottir, H. Håkansson, Pierre, J. M. Baumer, E. DiSalvo, C. and Sengers, P. (2012). Sustainability unpersuaded: how persuasion narrows our vision of sustainability. CHI 2012, Proceedings of the SIGCHI Conference on Human Factors in Computing Systems. Pp 947-956. ACM, NY, USA.

[38] Koomey, J.G., C. Calwell, J. Laitner, J. Thornton, R. Brown, J.H. Eto, C. Webber, and C. Cullicott. (2002). Sorry, wrong number: the use and misuse of numerical facts in analysis and media reporting of energy issues. Annual Review of Energy and the Environment, 27: 119-158.
[39] Ross, S. Evans, D and M. Webber. (2002). How LCA studies deal with uncertainty. International Journal of LCA 7(1) 47-52

[40] Henthorne, T. (2003). Cyber-Utopias: The Politics and Ideology of Computer Games. Studies in Popular Culture, 63-76.

[41] Ritzer, G. (1983). The "McDonaldization" of society. Journal of American Culture, 6(1), 100-107.

[42] Zapico, J.L., Turpeinen, M., Brandt, N. (2010). Greenalytics: A tool for mash-up life cycle assessment of websites. In Proceedings of the 24th International Conference on Informatics for Environmental Protection. Shaker Verlag, Aachen, Germany

[43] Hilty, L.M. Behrendt, S. Binswanger, M. Bruinink, A. Erdmann, L. Freohlich, J. Koehler, A. Kuster, N. Som, C. and Wuertenberger, F. (2005). The precautionary principle in the information society - Second edition. Report of the Centre for Technology Assessment. TA SWISS.

[44] Berkhout, P.H.G. Muskens, J.C. And Velthuijsen, J.W. (2000). Defining the rebound effect. Energy Policy 28, 425-432

[45] Allenby, B. (2006). The ontologies of industrial ecology? Progress in Industrial Ecology 3(1/2): 28-40

[46] Princen, T. (2003). Principles for Sustainability: From Cooperation and Efficiency to Sufficiency . Global Environmental Politics, 3(1), pp. 33-50. The MIT Press.

[47] Finnveden, G. (2000). On the Limitations of Life Cycle Assessment and Environmental System Analysis Tools in General. International Journal of LCA 5(4), 229-238.

[48] Borges, J.L (1975) A Universal History of Infamy. Penguin Books, London, 1975. ISBN 0-14-003959-7.

[49] De Saint-Exupéry, A. (1946). Le petit prince. Éditions Gallimard, Paris. 